VOL 4 (2020) NO 3

e-ISSN : 2549-9904

ISSN : 2549-9610

\title{
Will Covid-19 cases in the World reach 4 million? a forecasting approach using SutteARIMA
}

\author{
Ansari Saleh Ahmar"**, R. Rusli** \\ \#Business School, Faculty of Economics and Business, Universitat de Barcelona, Spain \\ "Department of Statistics, Faculty of Mathematics and Natural Science, Universitas Negeri Makassar, Indonesia \\ E-mail: ansarisaleh@unm.ac.id \\ ${ }^{* *}$ Department of Mathematics, Faculty of Mathematics and Natural Science, Universitas Negeri Makassar, Indonesia \\ E-mail: rusli.siman@unm.ac.id
}

\begin{abstract}
This study aimed to determine whether COVID-19 cases in the world would have reached 4 million cases with the SutteARIMA method forecasting approach. Data from this study were obtained from the Worldometer data from 01 March 2020 to 05 May 2020. Data were used to perform data fitting from 01 March 2020 to 28 April 2020 (29 April 2020 - 05 May 2020 ). The data fitting is used to examine the extent of the accuracy of the SutteARIMA method in predicting the data. To examine the level of the data accuracy, the MAPE method was used in this study. Results of forecasting data for the period of 29 April 2020 to 05 May 2020 : 72,$731 ; 84,666 ; 92,297 ; 100,797 ; 84,312 ; 81,517 ; 74845$. The accuracy of SutteARIMA for the period of 30 April 2020 - 06 May 2020 was $0.069 \%$. The forecasting results that had been obtained were 4 million cases, namely from 08 May 2020 to 10 May 2020 : $3,966,786 ; 4,047,328$ and $4,127,747$. The SutteARIMA method predicted that 4 million cases of COVID-19 in the world will be reported on the WHO situation report on day 110/111 or 09 May 2020/10 May 2020.
\end{abstract}

Keywords-COVID-19; short-term forecast; SutteARIMA.

\section{INTRODUCTION}

The first confirmed cases of COVID-19 in the world had been reported in Wuhan, China, and COVID-19 is an infectious disease caused by a new coronavirus (SARSCoV-2) discovered in China [1]. Based on the data submitted by Worldometer on 05 May 2020, the number of confirmed cases of COVID-19 in the world was $3,724,518$ or 81,247 cases from yesterday (04 May 2020) [2].

Forecasting is a method to determine the progress of case every day. Forecasting is used as the process of making predictions of the future. The results of this forecast can be used as a reference in the decision-making process. In the case of COVID-19, forecasting can be used as a means of looking at trends in the future and can provide input or advice to decision-makers to prevent the transmission of the COVID-19 case. Forecasting about the number of COVID19 cases had been discussed by Koczkodaj, et. al. using the Simple Heuristics (exponential curve) method [3]. Koczkodaj, et. al. predicted that 1 million COVID-19 cases outside China would be obtained on 30 March 2020 and this is different from the data displayed by the Worldometer on 03 April 2020. Based on this results, the authors forecasted the SutteARIMA method based on the forecasting results which have a high level of accuracy by referring to the forecasting results in the COVID-19 case in Spain [4][5].

\section{SUTTEARIMA}

SutteARIMA is a method of forecasting that combines the method of ARIMA [6] with the method of $\alpha$-Sutte Indicator [7].

The general form of the $\operatorname{ARIMA}(p, d, q)[6]$ :

$$
\begin{aligned}
& \phi_{p}(B) Z_{t}=\theta_{q}(B) a_{t}, a_{t} \sim W N\left(0, \sigma^{2}\right), \phi_{p}, \theta_{q} \in Z, t \in Z . \\
& \text { with } \phi_{p}(B)=\left(1-\phi_{1} B-\phi_{2} B^{2}-\ldots-\phi_{p} B^{p}\right) \quad(\text { for } \operatorname{AR}(\mathrm{p})) \\
& \text { and } \theta_{q}(B)=\left(1-\theta_{1} B-\theta_{2} B^{2}-\ldots-\theta_{q} B^{q}\right) \quad(\text { for MA(q) })
\end{aligned}
$$

If there is a differencing then the ARIMA model becomes as follows:

$\phi_{p}(B)(1-B)^{d} Z_{t}=\theta_{q}(B) a_{t}, a_{t} \sim W N\left(0, \sigma^{2}\right), \phi_{p}, \theta_{q} \in Z, t \in Z$.

with $\phi_{p}(B)=\left(1-\phi_{1} B-\phi_{2} B^{2}-\ldots-\phi_{p} B^{p}\right) \quad($ for $\operatorname{AR}(\mathrm{p})), \quad(1-B)^{d}$ (for differencing non seasonal) and $\theta_{q}(B)=\left(1-\theta_{1} B-\theta_{2} B^{2}-\ldots-\theta_{q} B^{q}\right.$ (for MA(q)). 
And the formula of the $\alpha$-Sutte Indicator method are as follows [8]:

$$
Z_{t}=\frac{\gamma\left(\frac{\Delta x}{\frac{\gamma+\delta}{2}}\right)+\beta\left(\frac{\frac{\Delta y}{\beta+\gamma}}{2}\right)+\alpha\left(\frac{\frac{\Delta z}{\alpha+\beta}}{2}\right)}{3}
$$

where:

$$
\begin{aligned}
& \delta=Z_{t-4} \\
& \gamma=Z_{t-3} \\
& \beta=Z_{t-2} \\
& \alpha=Z_{t-1} \\
& \Delta x=\gamma-\delta=Z_{t-3}-Z_{t-4} \\
& \Delta y=\beta-\gamma=Z_{t-2}-Z_{t-3} \\
& \Delta z=\alpha-\beta=Z_{t-1}-Z_{t-2} \\
& Z_{t}=\text { data at } t \text { time } \\
& Z_{t-k}=\text { data at }(t-k) \text { time }
\end{aligned}
$$

The equation (1), can be described as:

$\left(1-\phi_{1} B-\phi_{2} B^{2}-\ldots-\phi_{p} B^{p}\right) Z_{t}=\left(1-\theta_{1} B-\theta_{2} B^{2}-\ldots-\theta_{q} B^{q}\right) a_{t}$

$Z_{t}-\phi_{1} B Z_{t}-\phi_{2} B^{2} Z_{t}-\ldots-\phi_{p} B^{p} Z_{t}=a_{t}-\theta_{1} B a_{t}-\theta_{2} B^{2} a_{t}-\ldots-\theta_{q} B^{q} a_{t}$

While equation (3), can be reduced by using backward shift operator $\left(B^{p} Z_{t}=Z_{t-p}\right)$ :

$Z_{t}-\phi_{1} Z_{t-1}-\phi_{2} Z_{t-2}-\ldots-\phi_{p} Z_{t-p}=a_{t}-\theta_{1} a_{t-1}-\theta_{2} a_{t-2}-\ldots-\theta_{q} a_{t-q}$

$Z_{t}=\phi_{1} Z_{t-1}+\phi_{2} Z_{t-2}+\ldots+\phi_{p} Z_{t-p}+a_{t}-\theta_{1} a_{t-1}-\theta_{2} a_{t-2}-\ldots-\theta_{q} a_{t-q}$

If we define:

$$
\begin{aligned}
& \delta=Z_{t-4} \\
& \gamma=Z_{t-3} \\
& \beta=Z_{t-2} \\
& \alpha=Z_{t-1}
\end{aligned}
$$

The equation (4):

$Z_{t}=\phi_{1} \alpha+\phi_{2} \beta+\phi_{3} \gamma+\phi_{4} \delta+\ldots+\phi_{p} Z_{t-p}+$

$a_{t}-\theta_{1} a_{t-1}-\theta_{2} a_{t-2}-\ldots-\theta_{q} a_{t-q}$

and the equation (2) can be simplified as:

$$
\begin{aligned}
& Z_{t}=\frac{\gamma\left(\frac{\Delta x}{\frac{\gamma+\delta}{2}}\right)+\beta\left(\frac{\Delta y}{\frac{\beta+\gamma}{2}}\right)+\alpha\left(\frac{\Delta z}{\frac{\alpha+\beta}{2}}\right)}{3} \\
& Z_{t}=\frac{\frac{\gamma \Delta x}{2}+\frac{\beta \Delta y}{\frac{\beta+\gamma}{2}}+\frac{\alpha \Delta z}{\frac{\alpha+\beta}{2}}}{Z_{t}=} \frac{\frac{\gamma \Delta x}{3 \gamma+3 \delta}}{2}+\frac{\beta \Delta y}{\frac{3 \beta+3 \gamma}{2}}+\frac{\alpha \Delta z}{\frac{3 \alpha+3 \beta}{2}} \\
& Z_{t}=\frac{2 \gamma \Delta x}{3 \gamma+3 \delta}+\frac{2 \beta \Delta y}{3 \beta+3 \gamma}+\frac{2 \alpha \Delta z}{3 \alpha+3 \beta}
\end{aligned}
$$

$$
Z_{t}=\gamma \frac{2 \Delta x}{3 \gamma+3 \delta}+\beta \frac{2 \Delta y}{3 \beta+3 \gamma}+\alpha \frac{2 \Delta z}{3 \alpha+3 \beta}
$$

Let, Equation (4) added with Equation (5), we will find the formula of SutteARIMA:

$$
\begin{aligned}
& 2 Z_{t}= \phi_{1} \alpha+\phi_{2} \beta+\phi_{3} \gamma+\phi_{4} \delta+\ldots+\phi_{p} Z_{t-p}+a_{t}- \\
& \theta_{1} a_{t-1}-\theta_{2} a_{t-2}-\ldots-\theta_{q} a_{t-q}+ \\
& \frac{2 \Delta x}{3 \gamma+3 \delta}+\beta \frac{2 \Delta y}{3 \beta+3 \gamma}+\alpha \frac{2 \Delta z}{3 \alpha+3 \beta} \\
& Z_{t}= \alpha\left(\frac{\phi_{1}}{2}+\frac{\Delta z}{3 \alpha+3 \beta}\right)+\beta\left(\frac{\phi_{3}}{2}+\frac{2 \Delta y}{3 \beta+3 \gamma}\right)+\gamma\left(\frac{\phi_{3}}{2}+\frac{2 \Delta x}{3 \gamma+3 \delta}\right)+ \\
& \frac{\phi_{4} \delta}{2}+\ldots+\frac{\phi_{p} Z_{t-p}}{2}+\frac{a_{t}}{2}-\frac{\theta_{1} a_{t-1}}{2}-\frac{\theta_{2} a_{t-2}}{2}-\ldots-\frac{\theta_{q} a_{t-q}}{2}
\end{aligned}
$$

where:

$$
\begin{aligned}
& \delta=Z_{t-4} \\
& \gamma=Z_{t-3} \\
& \beta=Z_{t-2} \\
& \alpha=Z_{t-1} \\
& \Delta x=\gamma-\delta=Z_{t-3}-Z_{t-4} \\
& \Delta y=\beta-\gamma=Z_{t-2}-Z_{t-3} \\
& \Delta z=\alpha-\beta=Z_{t-1}-Z_{t-2} \\
& Z_{t}=\text { data at } t \text { time } \\
& Z_{t-p}=\text { data at }(t-p) \text { time }
\end{aligned}
$$

\section{SUTTEARIMA FORECAST}

The data for the COVID-19 cases were obtained from the Worldometer data. Data starts from 01 March 2020 - 05 May 2020. The total cases in the world are shown in Figure 1.

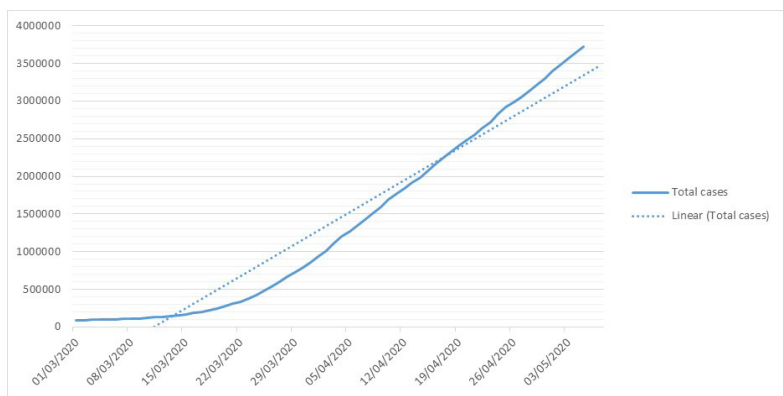

(a)

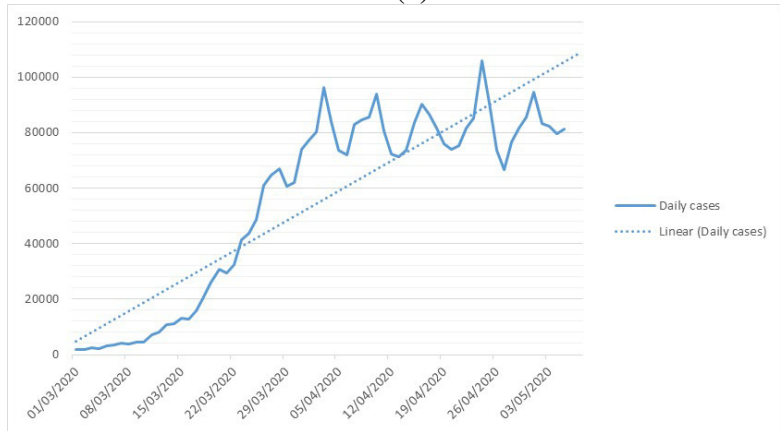

(b)

Fig. 1 (a) Total cases and linear trends (Total cases) of COVID-19 in the world (01 March 2020 - 05 May 2020); (b) Daily cases of COVID-19 in the world (01 March 2020 - 05 May 2020) 
On the base of figure 1, it can be seen that the COVID-19 case has an upward trend from time to time. This trend indicates that 4 million cases will be completed in a short time. This is also reinforced by Figure 2, which shows that the average number of confirmed cases per day from 22 April 2020 to 05 May 2020 is around 80,000 cases.

To further examine the likelihood of achieving the 4 million confirmed cases in the world, it is necessary to forecast the data. The forecasting method used in this study was the SutteARIMA method. The first stage carried out in this study was to determine the level of forecasting accuracy. Testing the accuracy of the SutteARIMA forecasting was done by fitting the data on the period of 29 April 2020 - 05 April 2020 by using the data from the period of 01 March 2020 - 28 April 2020. The level of forecasting accuracy can be seen from the mean absolute percentage error (MAPE). The results of this forecast are shown in Table 1.

TABLE 1

RESULTS OF FITTING CONFIRMED CASES OF COVID-19 IN WORLD

\begin{tabular}{|l|l|l|l|}
\hline Date & Actual & Forecast & APE \\
\hline $\mathbf{2 9 / 0 4 / 2 0 2 0}$ & 81648 & 72731 & 0,109 \\
\hline $\mathbf{3 0 / 0 4 / 2 0 2 0}$ & 85643 & 84666 & 0,011 \\
\hline $\mathbf{0 1 / 0 5 / 2 0 2 0}$ & 94550 & 92297 & 0,024 \\
\hline $\mathbf{0 2 / 0 5 / 2 0 2 0}$ & 83335 & 100797 & 0,210 \\
\hline $\mathbf{0 3 / 0 5 / 2 0 2 0}$ & 82260 & 84312 & 0,025 \\
\hline $\mathbf{0 4 / 0 5 / 2 0 2 0}$ & 79582 & 81517 & 0,024 \\
\hline $\mathbf{0 5 / 0 5 / 2 0 2 0}$ & 81247 & 74845 & 0,079 \\
\hline & & MAPE & 0,069 \\
\hline
\end{tabular}

Table 1 shows that the accuracy of the SutteARIMA method is $0.069 \%$ for the period of 29 April 2020 - 05 April 2020. By looking at the value of the low level of accuracy of the SutteARIMA method, the SutteARIMA method is used to predict when World cases reach 4 million cases and the results of this forecast can be seen in Table 2 .

TABLE 2

FORECAST 4 MILLION CASES OF COVID-19 IN WORLD

\begin{tabular}{|l|l|}
\hline Date & Forecast \\
\hline $\mathbf{0 8} / \mathbf{0 5} / \mathbf{2 0 2 0}$ & 3966786 \\
\hline $\mathbf{0 9 / 0 5 / 2 0 2 0}$ & 4047328 \\
\hline $\mathbf{1 0 / 0 5 / 2 0 2 0}$ & 4127747 \\
\hline
\end{tabular}

According to Table 2, the SutteARIMA method predicts that 4 million cases of COVID-19 in the world will be reported by the WHO situation report on 110/111 day or by 09 May 2020/10 May 2020.

\section{CONCLUSIONS}

The SutteARIMA method is a combination of the ARIMA method and the $\alpha$-Sutte indicator. In this study, the SutteARIMA method had an accuracy rate on data fitting of $0.069 \%$ or may be extended to $1 \%$. Based on the results of this study, the SutteARIMA method predicted that 4 million cases will be obtained from the WHO situation report on day $110 / 111$ or 09 May 2020 or 10 May 2020. It is expected that the results of this study will provide input to the World Government to be able to adopt policies aimed at preventing the COVID-19 cases, because based on the results of the ongoing analysis of the SutteARIMA method, if the addition of these cases is stagnant, it is expected that there will be around 4.5 million cases between 14 May 2020 and 15 May 2020 .

\section{REFERENCES}

[1] Y. Yang, F. Peng, R. Wang, K. Guan, T. Jiang, G. Xu, J. Sun, C. Chang, The deadly coronaviruses: The 2003 SARS pandemic and the 2020 novel coronavirus epidemic in China, J. Autoimmun. (2020) 102434. https://doi.org/10.1016/j.jaut.2020.102434

[2] Worldometer, Spain Coronavirus, (2020). https://www.worldometers.info/coronavirus/country/spain/ (accessed April 8, 2020).

[3] W.W. Koczkodaj, M.A. Mansournia, W. Pedrycz, A. WolnyDominiak, P.F. Zabrodskii, D. Strzaška, T. Armstrong, A.H. Zolfaghari, M. Debski, J. Mazurek, 1,000,000 cases of COVID-19 outside of China: The date predicted by a simple heuristic, Glob. Epidemiol. (2020) 100023. https://doi.org/10.1016/j.gloepi.2020.100023.

[4] A.S. Ahmar, E. Boj, SutteARIMA: Short-term forecasting method, a case: Covid-19 and stock market in Spain, Sci. Total Environ. (2020) 138883. https://doi.org/10.1016/j.scitotenv.2020.138883.

[5] A.S. Ahmar, E. Boj, The date predicted 200.000 cases of Covid-19 in Spain, J. Appl. Sci. Eng. Technol. Educ. 2 (2020). https://doi.org/10.35877/454RI.asci22102.

[6] W.W.S. Wei, Time Series Analysis: Univariate and Multivariate Methods, Addison-Wesley Publishing Company, New York, 1994.

[7] A.S. Ahmar, A. Rahman, U. Mulbar, $\alpha$-Sutte Indicator: A new method for time series forecasting, in: J. Phys. Conf. Ser., 2018. https://doi.org/10.1088/1742-6596/1040/1/012018.

[8] A.S. Ahmar, A Comparison of $\alpha$-Sutte Indicator and ARIMA Methods in Renewable Energy Forecasting in Indonesia, Int. J. Eng. Technol. 7 (2018) 20-22. 